Supplement of Biogeosciences, 14, 3957-3969, 2017

https://doi.org/10.5194/bg-14-3957-2017-supplement

(C) Author(s) 2017. This work is distributed under

the Creative Commons Attribution 3.0 License.

(c) (1)

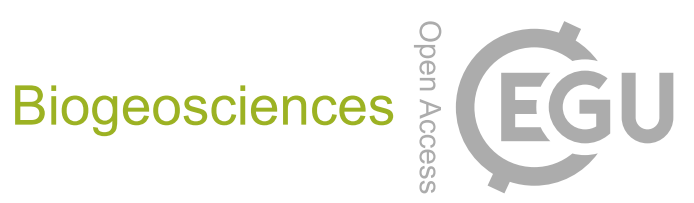

Supplement of

\title{
Recovery of biological soil crust richness and cover 12-16 years after wildfires in Idaho, USA
}

Heather T. Root et al.

Correspondence to: Heather T. Root (heatherroot@weber.edu)

The copyright of individual parts of the supplement might differ from the CC BY 3.0 License. 


\begin{tabular}{|c|c|c|c|c|c|c|c|c|}
\hline Plot & Site & BSC cover & BSC Freq & $\mathrm{BSC}$ rich & lat & long & avgdph & TRMT \\
\hline FN1 & Fenster & 1.05 & 36 & 11 & 45.26087 & -113.937 & 6.16 & 6 Burned \\
\hline FN10 & Fenster & 0.55 & 39 & 14 & 45.25004 & -113.931 & 6.29 & 9 Unburned \\
\hline FN11 & Fenster & 12.5 & 52 & 18 & 45.25125 & -113.926 & 6.24 & 4 Unburned \\
\hline FN12 & Fenster & 8.625 & 67 & 24 & 45.25099 & -113.926 & 6.52 & 2 Unburned \\
\hline FN2 & Fenster & 0.0625 & 22 & 10 & 45.25888 & -113.933 & 6.46 & 6 Burned \\
\hline FN21 & Fenster & 4.5125 & 51 & 23 & 45.25749 & -113.916 & 7.79 & 9 Unburned \\
\hline FN22 & Fenster & 11.375 & 63 & 20 & 45.25965 & -113.919 & 6.07 & 7 Burned \\
\hline FN4 & Fenster & 0.675 & 39 & 14 & 45.26152 & -113.934 & 6.7 & 7 Burned \\
\hline FN5 & Fenster & 0.9125 & 37 & 8 & 45.26142 & -113.937 & 6.15 & 5 Burned \\
\hline FN6 & Fenster & 1.0375 & 46 & 13 & 45.25995 & -113.933 & 6.46 & 6 Burned \\
\hline FN7 & Fenster & 6.25 & 61 & 18 & 45.2522 & -113.929 & 6.13 & 3 Unburned \\
\hline FN9 & Fenster & 7.925 & 24 & 12 & 45.24984 & -113.93 & 6.77 & 7 Unburned \\
\hline TB10 & Table Mtn & 0.1375 & 2 & 2 & 44.69925 & -114.203 & 7.25 & 5 Burned \\
\hline TB12 & Table Mtn & 3.75 & 63 & 26 & 44.69436 & -114.213 & 7.02 & 2 Unburned \\
\hline TB13 & Table Mtn & 1.2875 & 39 & 15 & 44.69117 & -114.211 & 6.3 & 3 Unburned \\
\hline TB15 & Table Mtn & 7.625 & 105 & 28 & 44.69065 & -114.212 & 7.5 & 5 Unburned \\
\hline TB17 & Table Mtn & 3.375 & 72 & 21 & 44.68883 & -114.208 & 6.61 & 1 Unburned \\
\hline TB18 & Table Mtn & 2.875 & 73 & 22 & 44.69217 & -114.206 & 7.19 & 9 Unburned \\
\hline TB2 & Table Mtn & 0 & 7 & 7 & 44.7013 & -114.204 & 6.91 & 1 Burned \\
\hline TB22 & Table Mtn & 2.5 & 89 & 28 & 44.69151 & -114.206 & 6.97 & 7 Unburned \\
\hline TB5 & Table Mtn & 0.2 & 16 & 10 & 44.70411 & -114.204 & 6.94 & 4 Burned \\
\hline TB6 & Table Mtn & 0.75 & 90 & 26 & 44.70337 & -114.208 & 6.68 & 8 Burned \\
\hline TB8 & Table Mtn & 2.875 & 69 & 21 & 44.70387 & -114.213 & 6.74 & 4 Burned \\
\hline TB9 & Table Mtn & 0.65 & 17 & 8 & 44.7014 & -114.203 & 6.65 & 5 Burned \\
\hline TO1 & Tobias & 0.05 & 11 & 5 & 44.75345 & -113.701 & 7.25 & 5 Burned \\
\hline T013 & Tobias & 14.625 & 84 & 23 & 44.76795 & -113.715 & 6.17 & 7 Burned \\
\hline T014 & Tobias & 3.8125 & 45 & 11 & 44.7683 & -113.714 & 6.64 & 4 Burned \\
\hline T015 & Tobias & 0.1 & 17 & 5 & 44.76951 & -113.712 & 5.98 & 8 Burned \\
\hline T016 & Tobias & 12.375 & 50 & 14 & 44.77044 & -113.723 & 6.89 & 9 Unburned \\
\hline T017 & Tobias & 1.025 & 37 & 14 & 44.76871 & -113.723 & & 1 Unburned \\
\hline T018 & Tobias & 5.6625 & 39 & 11 & 44.76836 & -113.72 & 6.04 & 4 Unburned \\
\hline TO2 & Tobias & 3.4 & 43 & 12 & 44.75291 & -113.698 & 6.48 & 8 Burned \\
\hline TO3 & Tobias & 0.95 & 16 & 5 & 44.75227 & -113.701 & 6.37 & 7 Burned \\
\hline TO4 & Tobias & 0.65 & 23 & 9 & 44.74039 & -113.706 & 6.46 & 6 Unburned \\
\hline TO5 & Tobias & 0.2125 & 20 & 7 & 44.7414 & -113.705 & 5.95 & 5 Unburned \\
\hline TO6 & Tobias & 1.4 & 30 & 13 & 44.73947 & -113.705 & 6.3 & 3 Unburned \\
\hline TX10 & Texas & 20.375 & 106 & 27 & 44.5797 & -113.292 & 6.11 & 1 Unburned \\
\hline TX13 & Texas & 1.2 & 79 & 20 & 44.5986 & -113.281 & 7.05 & 5 Burned \\
\hline TX14 & Texas & 0.0875 & 19 & 9 & 44.6012 & -113.279 & 7.33 & 3 Burned \\
\hline TX15 & Texas & 0.675 & 71 & 28 & 44.5993 & -113.288 & 7.07 & 7 Burned \\
\hline TX16 & Texas & 0.3375 & 56 & 19 & 44.5959 & -113.284 & 7.54 & 4 Burned \\
\hline TX17 & Texas & 0.1 & 31 & 12 & 44.5946 & -113.279 & 7.26 & 6 Burned \\
\hline TX19 & Texas & 13.125 & 111 & 27 & 44.6154 & -113.287 & 7.1 & 1 Unburned \\
\hline TX20 & Texas & 18 & 112 & 27 & 44.6099 & -113.285 & 6.94 & 4 Unburned \\
\hline TX21 & Texas & 22 & 120 & 34 & 44.6095 & -113.287 & 6.79 & 9 Unburned \\
\hline TX22 & Texas & 10.625 & 63 & 21 & 44.6123 & -113.292 & 7.27 & 7 Unburned \\
\hline
\end{tabular}




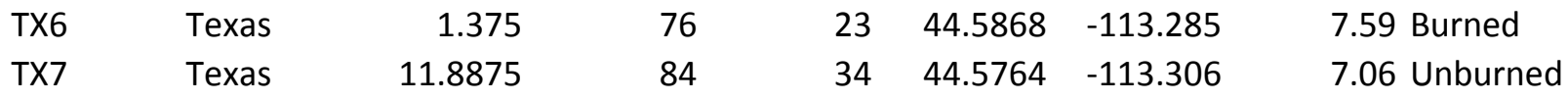




\begin{tabular}{|c|c|c|c|c|c|c|c|}
\hline Elev_M & rdnbr & dnbr & fire.level.cover & fire.level.richness & Foliose & Fruticose & SmFoliose \\
\hline 1669 & 1039 & 152 & 6.727083333 & 18.16666667 & 1 & 0 & 1 \\
\hline 1634 & 588 & 68 & 6.727083333 & 18.16666667 & 0 & 0 & 3 \\
\hline 1515 & 358 & 36 & 6.727083333 & 18.16666667 & 2 & 0 & 3 \\
\hline 1557 & 173 & -6 & 6.727083333 & 18.16666667 & 4 & 0 & 0 \\
\hline 1566 & 815 & 148 & 6.727083333 & 18.16666667 & 1 & 0 & 1 \\
\hline 1363 & 35 & -13 & 6.727083333 & 18.16666667 & 2 & 3 & 1 \\
\hline 1380 & 694 & 105 & 6.727083333 & 18.16666667 & 8 & 0 & 6 \\
\hline 1586 & 278 & 46 & 6.727083333 & 18.16666667 & 2 & 0 & 0 \\
\hline 1645 & 2124 & 293 & 6.727083333 & 18.16666667 & 1 & 0 & 0 \\
\hline 1654 & 2629 & 263 & 6.727083333 & 18.16666667 & 1 & 0 & 2 \\
\hline 1613 & -52 & -32 & 6.727083333 & 18.16666667 & 7 & 0 & 3 \\
\hline 1603 & -8 & -28 & 6.727083333 & 18.16666667 & 0 & 0 & 0 \\
\hline 2072 & 640 & 92 & 3.56875 & 23.33333333 & 0 & 0 & 0 \\
\hline 1977 & 19 & 12 & 3.56875 & 23.33333333 & 2 & 0 & 2 \\
\hline 1967 & -44 & 0 & 3.56875 & 23.33333333 & 3 & 0 & 3 \\
\hline 1975 & -63 & -6 & 3.56875 & 23.33333333 & 8 & 4 & 3 \\
\hline 2071 & -7 & 36 & 3.56875 & 23.33333333 & 1 & 5 & 7 \\
\hline 2003 & 142 & 29 & 3.56875 & 23.33333333 & 0 & 0 & 3 \\
\hline 2110 & 236 & 66 & 3.56875 & 23.33333333 & 0 & 0 & 0 \\
\hline 2025 & 62 & 21 & 3.56875 & 23.33333333 & 1 & 0 & 7 \\
\hline 2093 & -94 & 8 & 3.56875 & 23.33333333 & 0 & 1 & 0 \\
\hline 2092 & 121 & 33 & 3.56875 & 23.33333333 & 2 & 8 & 4 \\
\hline 2043 & 103 & 43 & 3.56875 & 23.33333333 & 6 & 0 & 2 \\
\hline 2119 & 324 & 69 & 3.56875 & 23.33333333 & 0 & 3 & 0 \\
\hline 1991 & 380 & 153 & 3.554166667 & 11.33333333 & 1 & 0 & 0 \\
\hline 1934 & 571 & 211 & 3.554166667 & 11.33333333 & 13 & 0 & 9 \\
\hline 1946 & 662 & 360 & 3.554166667 & 11.33333333 & 4 & 0 & 3 \\
\hline 1923 & 674 & 336 & 3.554166667 & 11.33333333 & 0 & 0 & 1 \\
\hline 1885 & 570 & 279 & 3.554166667 & 11.33333333 & 6 & 0 & 4 \\
\hline 1937 & 574 & 381 & 3.554166667 & 11.33333333 & 3 & 0 & 1 \\
\hline 1946 & 588 & 337 & 3.554166667 & 11.33333333 & 2 & 0 & 3 \\
\hline 1999 & 407 & 235 & 3.554166667 & 11.33333333 & 5 & 0 & 5 \\
\hline 2006 & 467 & 257 & 3.554166667 & 11.33333333 & 1 & 0 & 0 \\
\hline 2074 & 333 & 209 & 3.554166667 & 11.33333333 & 1 & 0 & 1 \\
\hline 2066 & 304 & 199 & 3.554166667 & 11.33333333 & 0 & 0 & 0 \\
\hline 2090 & 240 & 151 & 3.554166667 & 11.33333333 & 3 & 0 & 0 \\
\hline 1963 & -90 & 31 & 16.00208333 & 28.33333333 & 10 & 7 & 7 \\
\hline 1928 & 528 & 182 & 16.00208333 & 28.33333333 & 1 & 7 & 4 \\
\hline 1924 & 482 & 104 & 16.00208333 & 28.33333333 & 0 & 2 & 0 \\
\hline 1929 & 698 & 187 & 16.00208333 & 28.33333333 & 1 & 4 & 4 \\
\hline 1929 & 490 & 142 & 16.00208333 & 28.33333333 & 1 & 4 & 2 \\
\hline 1936 & 639 & 154 & 16.00208333 & 28.33333333 & 0 & 5 & 0 \\
\hline 1914 & -191 & 5 & 16.00208333 & 28.33333333 & 9 & 7 & 6 \\
\hline 1914 & 254 & 120 & 16.00208333 & 28.33333333 & 8 & 7 & 6 \\
\hline 1914 & -53 & 36 & 16.00208333 & 28.33333333 & 11 & 6 & 7 \\
\hline 1909 & -227 & 9 & 16.00208333 & 28.33333333 & 1 & 6 & 5 \\
\hline
\end{tabular}


$\begin{array}{lllll}1945 & 603 & 194 & 16.00208333 & 28.33333333\end{array}$

2

1971

$31 \quad 16.00208333$

28.33333333

6

3 


\begin{tabular}{|c|c|c|c|c|c|c|c|c|c|}
\hline Squamulos & custc & sto & & Cushion & Mat & & & TurfTall & AnnGrass \\
\hline 4 & 1 & 0 & 0 & & 0 & 0 & 20 & 9 & 2.5 \\
\hline 11 & 1 & 2 & 0 & & 0 & 0 & 15 & 7 & 9.6375 \\
\hline 8 & 2 & 5 & 0 & & 0 & 3 & 21 & 8 & 1.75 \\
\hline 10 & 15 & 5 & 0 & & 0 & 2 & 23 & 8 & 8 \\
\hline 3 & 1 & 0 & 0 & & 0 & 0 & 13 & 5 & 8.875 \\
\hline 10 & 9 & 13 & 0 & & 1 & 0 & 3 & 11 & 5.7625 \\
\hline 11 & 7 & 3 & 0 & & 0 & 5 & 16 & 7 & 7 \\
\hline 4 & 0 & 5 & 0 & & 0 & 1 & 19 & 8 & 4.125 \\
\hline 2 & 0 & 0 & 0 & & 0 & 0 & 26 & 8 & 2.125 \\
\hline 8 & 0 & 4 & 0 & & 0 & 0 & 22 & 9 & c \\
\hline 10 & 4 & 5 & 0 & & 0 & 2 & 22 & 8 & 0 \\
\hline 6 & 3 & 2 & 0 & & 1 & 0 & 6 & 8 & 13.625 \\
\hline 0 & 0 & 0 & 0 & & 0 & 0 & 1 & 1 & 1 \\
\hline 15 & 5 & 23 & 0 & & 0 & 0 & 7 & 9 & c \\
\hline 6 & 1 & 9 & 0 & & 0 & 0 & 12 & 5 & 0 \\
\hline 23 & 16 & 34 & 0 & & 0 & 0 & 9 & 8 & 0 \\
\hline 15 & 4 & 23 & 0 & & 0 & 0 & 9 & 8 & 8 \\
\hline 22 & 3 & 28 & 0 & & 0 & 0 & 9 & 8 & 3 \\
\hline 0 & 0 & 7 & 0 & & 0 & 0 & 0 & 0 & D \\
\hline 28 & 7 & 31 & 0 & & 0 & 0 & 7 & 8 & 3 \\
\hline 1 & 0 & 4 & 0 & & 0 & 0 & 3 & 7 & 7 \\
\hline 18 & 6 & 30 & 0 & & 0 & 0 & 15 & 7 & 0 \\
\hline 18 & 7 & 20 & 0 & & 0 & 0 & 14 & 2 & 2 \\
\hline 0 & 1 & 10 & 0 & & 0 & 0 & 0 & 3 & 3 \\
\hline 0 & 0 & 0 & 0 & & 0 & 0 & 9 & 1 & 1 \\
\hline 11 & 5 & 13 & 0 & & 0 & 0 & 25 & 8 & 0 \\
\hline 5 & 1 & 3 & 0 & & 0 & 0 & 21 & 8 & 8 \\
\hline 0 & 0 & 0 & 0 & & 0 & 0 & 13 & 3 & 0.625 \\
\hline 7 & 0 & 7 & 0 & & 0 & 1 & 16 & 9 & $\theta$ \\
\hline 1 & 0 & 0 & 0 & & 0 & 5 & 20 & 7 & 7 \\
\hline 5 & 0 & 3 & 0 & & 0 & 0 & 20 & 6 & 5 \\
\hline 5 & 0 & 0 & 0 & & 0 & 1 & 17 & 10 & 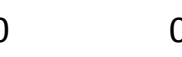 \\
\hline 0 & 0 & 0 & 0 & & 0 & 0 & 14 & 1 & 0.125 \\
\hline 1 & 0 & 0 & 0 & & 0 & 0 & 15 & 5 & 0.125 \\
\hline 1 & 0 & 0 & 0 & & 0 & 3 & 15 & 1 & 3.3875 \\
\hline 4 & 0 & 0 & 0 & & 0 & 1 & 18 & 4 & 0 \\
\hline 19 & 7 & 26 & 16 & & 0 & 0 & 9 & 11 & 1 \\
\hline 15 & 9 & 19 & 11 & & 0 & 0 & 10 & 9 & 9 \\
\hline 1 & 2 & 2 & 2 & & 0 & 0 & 15 & 3 & c \\
\hline 15 & 9 & 20 & 8 & & 0 & 0 & 12 & 4 & 4 \\
\hline 12 & 6 & 13 & 12 & & 0 & 0 & 2 & 4 & 4 \\
\hline 2 & 1 & 8 & 8 & & 0 & 0 & 8 & 3 & 0 \\
\hline 26 & 12 & 22 & 18 & & 0 & 0 & 5 & 8 & 8 \\
\hline 22 & 11 & 20 & 19 & & 0 & 0 & 11 & 12 & 2 \\
\hline 26 & 10 & 22 & 21 & & 0 & 0 & 14 & 11 & 1 \\
\hline 5 & 5 & 18 & 10 & & 0 & 0 & 4 & 11 & 1 \\
\hline
\end{tabular}




$\begin{array}{rrrrrrrrr}9 & 7 & 26 & 7 & 0 & 0 & 22 & 8 & 0 \\ 15 & 8 & 27 & 8 & 0 & 0 & 4 & 8 & 0\end{array}$




\begin{tabular}{|c|c|c|c|c|c|c|c|c|}
\hline Cowpie & feces & Forb & Litter & PerGrass & Rock & Shrub & Soil & Wood \\
\hline 0 & 0 & 9.7375 & 0 & 15.75 & 14.5 & 36.125 & 28.625 & 0.0125 \\
\hline 0 & 0.125 & 12.675 & 0 & 14.25 & 30.625 & 12.625 & 31.25 & 0 \\
\hline 2 & 0.625 & 8.2625 & 0 & 27.65 & 9.5 & 36.5 & 19 & 0 \\
\hline 0.375 & 0.25 & 6.125 & 0 & 23.75 & 12.625 & 11.25 & 32.875 & 1.5 \\
\hline 0 & 0 & 5.6375 & 0 & 6.5 & 36.375 & 23.875 & 17.875 & 0.0125 \\
\hline 0 & 0 & 8.7 & 0 & 17.5125 & 36.7625 & 5 & 17.3875 & 0 \\
\hline 0.375 & 0 & 13.5 & 0 & 39.375 & 21 & 2.25 & 17.125 & 0 \\
\hline 0.125 & 0 & 14.1 & 0 & 24.75 & 11.5 & 35.375 & 34.5 & 1.5 \\
\hline 0.25 & 0 & 8.775 & 0 & 15.875 & 14.5 & 35.25 & 39.125 & 0 \\
\hline 0.875 & 0.375 & 18.6125 & 0 & 26.0125 & 5.875 & 34.375 & 30.125 & 0.25 \\
\hline 1.125 & 0.75 & 13.0125 & 0 & 44.5 & 13.5 & 7.5 & 15.6375 & 0 \\
\hline 0 & 0 & 3.5625 & 0 & 5.125 & 54.875 & 12.875 & 18.5 & 2.75 \\
\hline 0.625 & 0 & 11.175 & 0 & 15.625 & 17.75 & 16.125 & 39.375 & 1 \\
\hline 1.25 & 0 & 9.3 & 0 & 14.625 & 24.375 & 25.875 & 30.75 & 1.875 \\
\hline 1.125 & 0 & 17.4375 & 0 & 17.65 & 6.875 & 26.875 & 18.625 & 2.5 \\
\hline 0.375 & 0 & 15.975 & 0 & 12.8125 & 27.875 & 10.125 & 27.875 & 0.125 \\
\hline 0 & 0 & 10.0375 & 0 & 31.625 & 16.75 & 23.25 & 18.25 & 0.625 \\
\hline 0.75 & 0 & 6.95 & 0 & 13.625 & 42 & 19.75 & 20 & 0 \\
\hline 0 & 0 & 9.925 & 0 & 9.625 & 30.625 & 26.125 & 25.625 & 0 \\
\hline 0.5 & 0 & 9.8625 & 0 & 17.75 & 27.125 & 20.125 & 28.75 & 0 \\
\hline 0.5 & 0 & 7.9375 & 0 & 18.125 & 20 & 26.25 & 22.5 & 2.875 \\
\hline 1.25 & 0 & 19.6625 & 0 & 21.875 & 24.125 & 13 & 24.625 & 0 \\
\hline 0 & 0 & 12.3625 & 0 & 22 & 25.375 & 17.25 & 22 & 0 \\
\hline 0.25 & 0 & 15.35 & 0 & 7.8875 & 28.125 & 25.5 & 23.125 & 0.625 \\
\hline 0.375 & 0 & 16.825 & 0 & 83.4 & 3.8125 & 2.125 & 7.0625 & 0 \\
\hline 0.0125 & 0 & 13.325 & 0 & 40.125 & 29.875 & 0 & 21.75 & 0.0125 \\
\hline 3.75 & 0 & 19.475 & 0 & 46.5 & 11.375 & 1.75 & 12.25 & 0.0125 \\
\hline 0 & 0 & 18.175 & 0 & 65.875 & 5.3 & 0.5 & 18.275 & 0.125 \\
\hline 0.25 & 0 & 39.0125 & 0 & 38.9 & 1.05 & 26.375 & 7.55 & 2.5 \\
\hline 0 & 0 & 31.2125 & 0 & 40.025 & 0.2125 & 42.25 & 2.05 & 0 \\
\hline 1.75 & 0 & 11.6625 & 0 & 53.6625 & 6.1375 & 1.2625 & 12.5125 & 0 \\
\hline 0 & 0 & 47.4375 & 0 & 50.625 & 11.375 & 7.375 & 8.875 & 0.5125 \\
\hline 0.75 & 0 & 22.675 & 0 & 32.15 & 6.125 & 19.375 & 21.375 & 0.75 \\
\hline 5.125 & 0 & 15.8625 & 0 & 85 & 0.875 & 0 & 3.5 & 0 \\
\hline 0 & 0 & 34.875 & 0 & 51.5375 & 4.75 & 5.625 & 12.3875 & 0 \\
\hline 0 & 0 & 32.0125 & 6.625 & 42.1625 & 3.875 & 25.25 & 2.5 & 0 \\
\hline 0 & 0 & 2.8125 & 0 & 8.6375 & 26 & 34.5 & 26.25 & 3.75 \\
\hline 1.25 & 0 & 2.425 & 0 & 31 & 25.875 & 8.25 & 30.625 & 0.375 \\
\hline 1.875 & 0 & 0 & 0 & 32.125 & 4.75 & 17.5 & 38 & 0.5 \\
\hline 1.25 & 0 & 1.575 & 0 & 37.25 & 38.5 & 2.7625 & 15.375 & 0.875 \\
\hline 1.375 & 0 & 0.4375 & 0 & 37.75 & 21 & 0.525 & 24.375 & 0.625 \\
\hline 3.375 & 0 & 5.7875 & 0 & 33.8875 & 10.875 & 0.125 & 30 & 0.5 \\
\hline 0 & 0 & 0.7875 & 0 & 25.3875 & 25.5 & 26.6375 & 30.125 & 0.625 \\
\hline 0.375 & 0 & 3.275 & 0 & 29.125 & 19.875 & 35.3875 & 23.375 & 0.125 \\
\hline 0 & 0 & 0.775 & 0 & 9.75 & 24.75 & 40.9 & 23.125 & 0 \\
\hline 2.875 & 0 & 0.75 & 0 & 19.5 & 29.25 & 34.875 & 20.625 & 0 \\
\hline
\end{tabular}




$\begin{array}{rrrrrrrrr}1 & 0 & 0.9625 & 0 & 33.9125 & 31.25 & 1.65 & 16.625 & 0 \\ 0 & 0 & 9.7 & 0 & 11.5375 & 20.25 & 27.6375 & 17.375 & 0\end{array}$




\begin{tabular}{|c|c|c|c|}
\hline CMD_at & PPT_sp & Tmin_wt & Tmin_sm \\
\hline 83 & 110 & -10.2 & 7.4 \\
\hline 89 & 98 & -10.4 & 7.4 \\
\hline 95 & 92 & -10 & 8 \\
\hline 93 & 92 & -10.1 & 7.8 \\
\hline 88 & 104 & -10 & 7.9 \\
\hline 101 & 82 & -9.4 & 8.9 \\
\hline 99 & 87 & -9.4 & 8.9 \\
\hline 86 & 106 & -10 & 7.8 \\
\hline 83 & 109 & -10.2 & 7.5 \\
\hline 85 & 103 & -10.2 & 7.5 \\
\hline 90 & 97 & -10.3 & 7.5 \\
\hline 91 & 96 & -10.3 & 7.5 \\
\hline 74 & 129 & -11 & 5.7 \\
\hline 78 & 126 & -10.9 & 6.1 \\
\hline 80 & 124 & -10.9 & 6.2 \\
\hline 80 & 123 & -11 & 6.1 \\
\hline 78 & 122 & -11.2 & 5.7 \\
\hline 79 & 124 & -11 & 6 \\
\hline 72 & 131 & -11.1 & 5.5 \\
\hline 79 & 124 & -11.1 & 5.9 \\
\hline 71 & 134 & -11 & 5.6 \\
\hline 71 & 135 & -10.9 & 5.6 \\
\hline 72 & 134 & -10.9 & 5.8 \\
\hline 72 & 131 & -11.1 & 5.5 \\
\hline 61 & 147 & -11.4 & 6.5 \\
\hline 65 & 136 & -10.9 & 7.2 \\
\hline 66 & 135 & -10.9 & 7.1 \\
\hline 68 & 133 & -10.8 & 7.2 \\
\hline 64 & 138 & -10.6 & 7.6 \\
\hline 62 & 139 & -10.8 & 7.3 \\
\hline 63 & 138 & -10.8 & 7.2 \\
\hline 60 & 148 & -11.4 & 6.6 \\
\hline 60 & 147 & -11.5 & 6.4 \\
\hline 55 & 147 & -11.7 & 6.1 \\
\hline 55 & 148 & -11.7 & 6.1 \\
\hline 54 & 148 & -11.8 & 6 \\
\hline 101 & 76 & -11.9 & 5.8 \\
\hline 104 & 71 & -11.9 & 5.9 \\
\hline 104 & 71 & -11.8 & 5.9 \\
\hline 104 & 71 & -12 & 5.8 \\
\hline 104 & 72 & -11.9 & 5.8 \\
\hline 103 & 72 & -11.8 & 5.9 \\
\hline 107 & 67 & -12.1 & 5.7 \\
\hline 106 & 69 & -12 & 5.8 \\
\hline 106 & 68 & -12 & 5.8 \\
\hline 107 & 67 & -12.1 & 5.7 \\
\hline
\end{tabular}


5.8

99

77

$-12$

5.5 